\title{
La web académica española en el contexto del Espacio Europeo de Educación Superior: estudio exploratorio
}

\author{
Por José-Luis Ortega e Isidro F. Aguillo
}

\begin{abstract}
Resumen: Se pretende representar visualmente la situación de la web académica española y su relación con el contexto del espacio web universitario europeo, con la finalidad de conocer su topología, sus universidades más destacadas y su posición respecto al resto de espacios web europeos. Para ello se han utilizado mapas de co-enlaces y grafos que han permitido visualizar las distintas redes y sus peculiaridades. Los resultados muestran que la red española se encuentra bastante aislada del resto de universidades europeas, ya que tanto el porcentaje de enlaces salientes como de entrantes son bajos con respecto al volumen de contenidos y al número de universidades que presenta. A la vez, la red académica española está bastante cohesionada entre sí, con un gran núcleo fuertemente interconectado. Creemos que el bajo uso del inglés y la escasa publicación electrónica de contenidos de calidad (repositorios) favorece este aislamiento, además de que las universidades españolas tienen un componente más local que europeísta.
\end{abstract}

Palabras clave: Cibermetría, Visualización de información, Análisis de redes, Universidades españolas.

\section{Title: Spain's academic web space in the Eu- ropean Higher Education Area: an exploratory approach}

Abstract: Spain's academic web space is diagrammed, together with its relationship with the European Higher Education Area (EHEA), with the goal of determining the topology, the outstanding universities, and our standing with respect to other European web spaces. Several co-link maps and network graphs visually represent these relationships among universities and web spaces. The results show that the Spanish network is rather isolated from other European universities, because the out-and inlink percentages are quite low considering the volume of content and the number of Spanish universities. On the other hand, Spain's academic web space is quite cohesive, with a significant nucleus that is strongly interconnected. We conclude that the limited use of the English language and minimal electronic publication of quality research in accessible repositories could explain this isolation. In addition, Spanish universities have a more local than international emphasis.

Keywords: Webometrics, Information visualization, Network analysis, Spanish universities.

Ortega, José-Luis; Aguillo, Isidro F. “La web académica española en el contexto del Espacio Europeo de Educación Superior: estudio exploratorio". En: El profesional de la información, 2007, septiembre-octubre, v. 16, n. 5, pp. 417-425.

DOI: 10.3145/epi.2007.sep.03

\section{Introducción}

España ha tenido una importancia clave en el desarrollo de la investigación sobre la web. Desde un punto de vista cibermétrico, puede ser considerado un país bastante prolífico en esta materia. Cabe destacar que uno de los trabajos pioneros de esta disciplina fue el artículo de Rodríguez Gairín (1997), donde analizaba la potencialidad de los buscadores, en concreto AltaVista, como una herramienta para valorar sedes web académicas al igual que el Science Citation Index lo hacía para la producción científica. Ese mismo año aparecía la revista Cybermetrics (Aguillo, 1997) especializada en estudios cuantitativos de la Red que consiguió asentar la disciplina y aglutinar a la comunidad científica internacional interesada en estos estudios. Por otro lado, diversos libros se han publicado en castellano describiendo metodologías y técnicas de análisis cibermé- 
tricos (Faba-Perez; Guerrero-Bote; Moya-Anegón, 2004; Alonso Berrocal, García Figuerola; Zazo Rodríguez, 2004). Por último, el grupo de Baeza-Yates ha realizado una profunda caracterización de la web española, describiendo su estructura topológica (BaezaYates; Castillo; López, 2005). Con respecto al análisis de la web académica en España, destacan los trabajos de Thelwall y Aguillo (2003) y Ortega (2007) desde un punto de vista cuantitativo y diversos trabajos desde el contexto cualitativo (Pinto Molina, et al., 2004; Térmens Graells; Ribera Turró; Sulé Duesa, 2003).

La visualización de la web como medio para comprender su estructura y relaciones ha sido una tarea compleja y llena de dificultades debido a la gran cantidad de contenidos y de enlaces que conectan zonas lejanas dentro de ella (Dodge, 2007; Paterson; Cox, 2007; Zook, 2000). La cibermetría ha intentado elaborar una representación gráfica a través de distintas técnicas. Polanco, Boudourides, Besagni y Roche (2001) mapearon y agruparon 791 dominios web universitarios europeos usando análisis de co-enlaces, y detectaron un grupo de universidades altamente enlazadas. Heimeriks, Horlesberger y Van Den Besselaar (2003) y Heimeriks (2005) representaron la red de enlaces entre 220 universidades de la Unión Europea al nivel de departamentos, universidades y países encontrando patrones culturales y lingüísticos en sus relaciones. Vaughan (2006) también usó análisis de co-enlaces para mostrar las universidades canadienses, agrupándolas por características lingüísticas.

\section{Objetivos}

En este artículo se pretende hacer un recorrido exploratorio sobre diversos mapas de la web académica española. Enmarcada dentro de la red europea, se busca describir su posición respecto al resto de Europa y sus características particulares. Por otro lado, se quieren detectar relaciones regionales y mostrar las universidades más importantes dentro de la red española y europea. Además, se pretende hacer un análisis de su estructura y topología. Para ello se usan diversas visualizaciones desde distintos puntos de vista y se aplican técnicas de análisis de redes sociales (ARS).

\section{Metodología}

El presente trabajo se enmarca dentro de un proyecto más amplio donde se analizan las relaciones de 535 universidades europeas a través de sus enlaces (Ortega; Aguillo; Cothey; Scharnhorst, 2008). Representan a las universidades europeas más destacadas por su visibilidad y cantidad de contenidos tomadas de las 1.000 primeras universidades del Ranking Mundial de Universidades en la Web (webometrics.info) (Aguillo; Granadino; Ortega; Prieto, 2006), que recoge un total de 3.690 universidades europeas. Seguidamente, a través de Yahoo! Search se extrajeron los enlaces salientes entre estos 535 centros con la siguiente estrategia:

\section{+ site: $\{$ dominio universitario $A\}+$ linkdomain: $\{$ dominio universitario $B\}$}

Esta cadena de búsqueda permite recuperar todos los enlaces salientes de un dominio web (site:) hacia otro (linkdomain:). Entendemos por enlace saliente todo aquel que desde un dominio A apunta a cualquiera de los dominios involucrados en el estudio. De igual forma, uno entrante es todo vínculo a un dominio web A desde el resto de dominios web analizados. Un enlace entrante permite valorar la visibilidad, ya que cuantos más posea una web dentro de una red mayor probabilidad existe de que sea visitada, mientras que un enlace saliente permite estimar la capacidad de una web para generar tráfico.

Yahoo! Search fue usado porque es el único buscador, junto con Live Search, que permite combinar operadores además de mostrar la totalidad de enlaces existentes en un dominio web. Sin embargo la cobertura del primero es mayor que la del segundo.

Durante el proceso de extracción no se pudieron obtener datos de la Universidad de Valencia (uv.es), ya que Yahoo! Search no poseía ninguna información sobre esta universidad (desconocemos las razones de esta situación) por lo que sólo se recogen sus enlaces entrantes. Por otro lado, los datos de universidades que poseían varios dominios (por ejemplo udg.es y udg.edu para la Universidad de Girona) fueron fusionados y se tomó el que más datos poseía.

Una vez obtenidos los datos se construyó una matriz de co-enlaces, calculadas a través del coseno de Salton y dimensionadas a través del escalamiento multidimensional (MDS) con el software SPSS 13 (Ortega; Aguillo, 2006). Este método fue utilizado porque los enlaces siguen una distribución potencial (power law) (Barabási; Albert; Jeong, 2000) y esta medida no se ve afectada por este hecho, cosa que sí sucede con la correlación, la cual se basa en la media de la distribución y su operatividad está en distribuciones normales o gaussianas. El MDS fue usado para obtener las coordenadas de los nodos en función del grado de similitud aportado por el coseno. Esta técnica se ha mostrado adecuada para detectar relaciones temáticas entre sedes web (Heimeriks; Van Den Besselaar, 2006; Ortega, et al., 2008).

Se añadieron distintos atributos a los gráficos para conseguir una mayor explicación: el color representa la nacionalidad en mapas europeos y regiones en el mapa español; el tamaño expresa la cantidad de páginas accesibles de cada dominio web; la forma muestra el tipo de universidad por criterios temáticos; y el grosor de los 


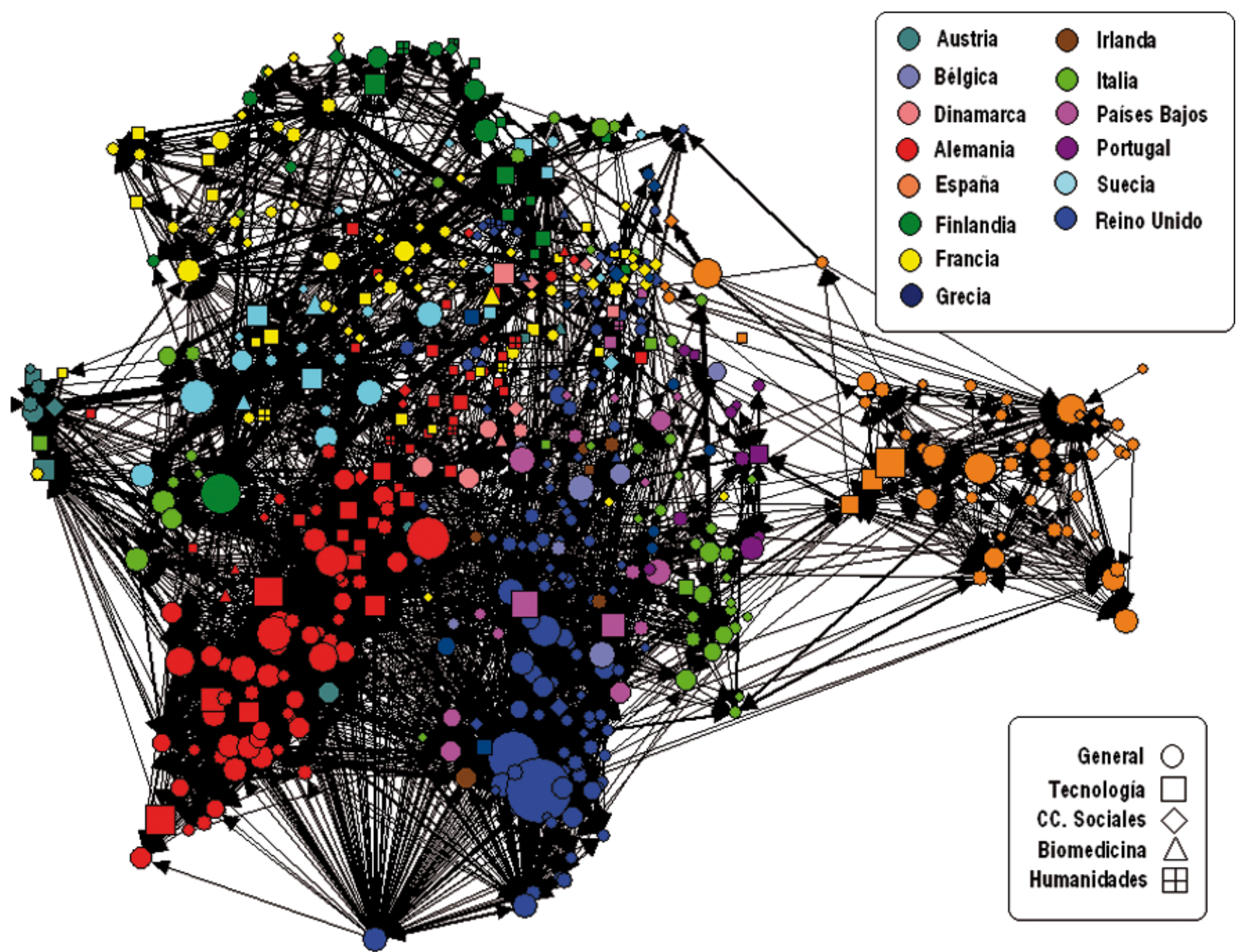

Figura 1. Mapa de co-enlaces de la red de universidades europeas ( $\varphi=0,086 ; 527$ nodos; $\operatorname{arcos} \geq 50$ enlaces; layout: coordenadas MDS)

arcos expresa la cantidad de enlaces que van de un dominio web a otro. Finalmente, se representaron los distintos gráficos a través del programa de representación de redes NetDraw 2.28. Las 50 universidades españolas que forman parte de esta muestra serán analizadas dentro de su contexto europeo.

Por último, se examinará la estructura y topología de la red académica española mediante $A R S$, un conjunto de técnicas de análisis para el estudio formal de las relaciones entre actores y para analizar las estructuras sociales que surgen de las mismas (Sanz Menéndez, 2003). Para el análisis de estas redes sociales, el ARS ha desarrollado un conjunto de categorías, procedimientos y algoritmos, que nos dan información sobre la estructura, tales como componentes, densidad, unipolaridad, integración y centralización. En nuestro caso son utilizados varios indicadores:

- Grado nodal (Degree): número de enlaces directos que recibe o realiza un nodo. Se puede hablar de grado de entrada (InDegree) o número de enlaces que recibe un nodo y grado de salida (OutDegree) o número de enlaces que salen de un nodo si nos referimos a una red dirigida como la web, esto es, una red cuyos enlaces sólo tienen un único sentido. Nos permite conocer qué función poseen distintos nodos en la integración y cohesión de una red. Esta medida es siempre normalizada como porcentaje de enlaces que tiene un nodo sobre el total de la red (Leydesdorff, 2007).

- Grado de intermediación (Betweenness): indica la frecuencia en que aparece un nodo en el camino más corto que conecta a otros dos (Nooy; Mrvar; Batagelj, 2005). Muestra cuando una sede web es intermediaria entre otras dos que no tienen enlaces directos entre sí.

- $k$-Cores: es un método que permite simplificar una red y localizar su núcleo (Seidman, 1983). Un $k$ Core es un grupo de nodos que entre todos tienen un grado nodal igual o mayor que $k$. Cuanto mayor sea el grado nodal mayor es la relación entre éstos y su densidad de enlaces. Si se simplifica la red al máximo, localizando el $k$-Core mayor posible, podemos identificar la parte de la red más densa y más altamente conectada entre sí.

\section{Resultados}

Respecto a su situación en Europa (figura 1), el mapa muestra que la web académica española está escasamente conectada con el resto de universidades eu- 


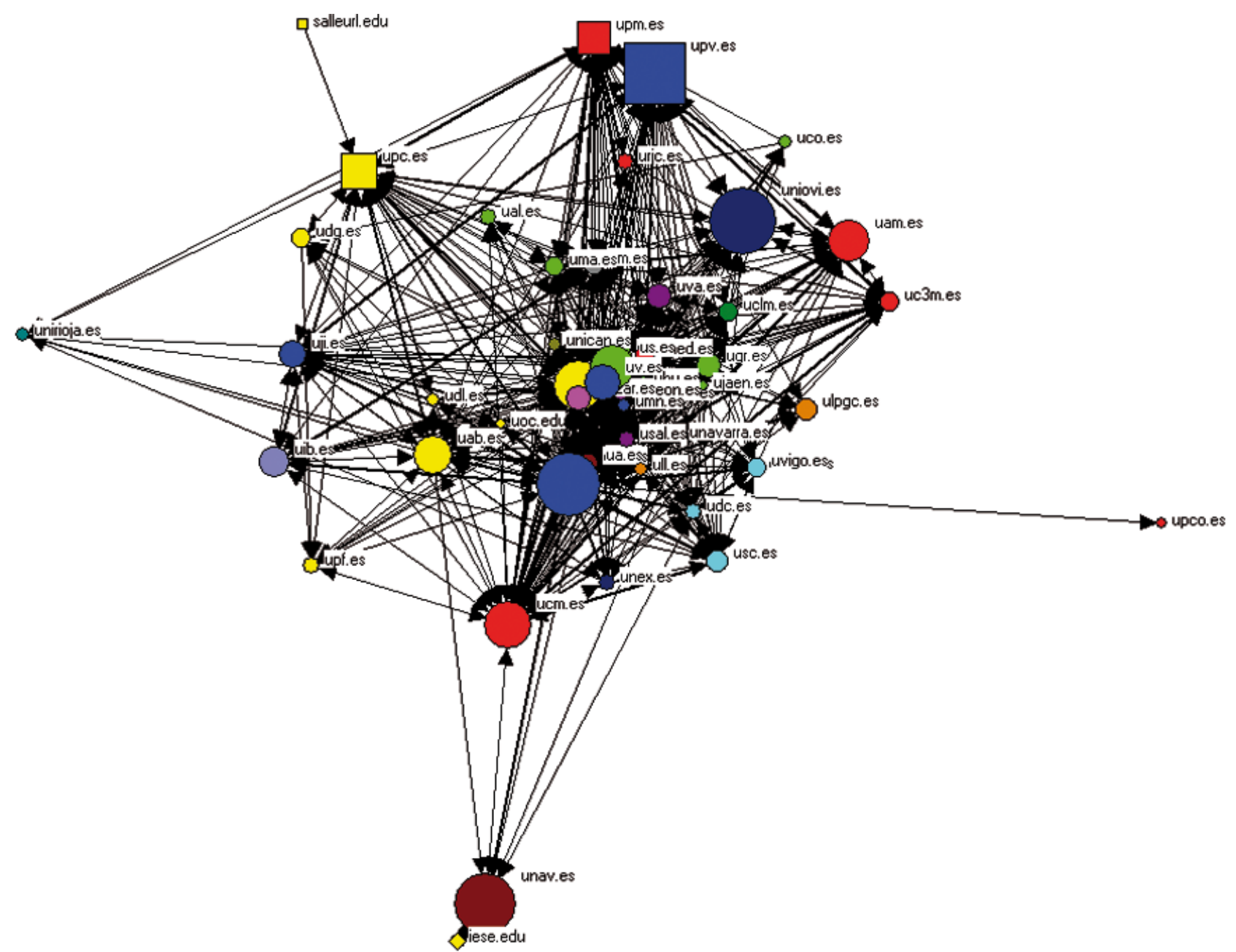

Figura 2. Mapa de co-enlaces de universidades españolas ( $\varphi=0,071 ; 50$ nodos; $\operatorname{arcos} \geq 50$ enlaces; layout: coordenadas MDS)

ropeas, debido a la baja tasa de co-enlaces que posee con la web académica europea. Sin embargo, se aprecia que la red española es compacta y densa. De esta forma se muestran síntomas de aislamiento con respecto a Europa, aunque también posee una fuerte cohesión a nivel nacional. Esto se puede deber a aspectos idiomáticos, ya que el uso de páginas en inglés por parte de las universidades españolas es bastante bajo con respecto al resto las europeas (Ortega; Aguillo, 2007). Por otro lado, se aprecia que el tamaño de las universidades españolas (número de páginas) es bastante bueno, equiparable al resto de universidades de los grandes países, a excepción de Francia.

\section{"La web académica española está escasamente conectada con el resto de universidades europeas"}

En el gráfico de las universidades españolas (figura 2), se distinguen ciertos grupos regionales como son las universidades catalanas (amarillo), las gallegas (azul

\begin{tabular}{|c|l|c|c|c|}
\hline & Universidad & URL & $\begin{array}{c}\text { Webs } \\
\text { enlazadas }\end{array}$ & \% \\
\hline 1 & $\begin{array}{l}\text { Universidad } \\
\text { Politécnica de } \\
\text { Madrid }\end{array}$ & upm.es & 15 & 42,86 \\
\hline 2 & $\begin{array}{l}\text { Universidad } \\
\text { Autónoma de } \\
\text { Madrid }\end{array}$ & uam.es & 11 & 31,42 \\
\hline 3 & $\begin{array}{l}\text { Universitat } \\
\text { Politècnica de } \\
\text { Catalunya }\end{array}$ & upc.es & 10 & 28,57 \\
\hline 4 & $\begin{array}{l}\text { Universitat de } \\
\text { Barcelona }\end{array}$ & ub.es & 9 & 25,71 \\
\hline 5 & $\begin{array}{l}\text { Universidad } \\
\text { Complutense } \\
\text { de Madrid }\end{array}$ & ucm.es & 7 & 20,00 \\
\hline Total & & 35 & \\
\hline
\end{tabular}

Tabla 1. Universidades españolas que enlazan a universidades europeas

celeste) o las andaluzas (verde claro). Cabe destacar el tamaño de la Universidad de Alicante (ua.es) o de la Oviedo (uniovi.es), debido al gran número de páginas que publican. Es significativa también la relación lingüística de las universidades de habla catalana perte- 


\begin{tabular}{|c|c|c|c|c|c|}
\hline Universidad & dominio & $\begin{array}{c}\text { nIn } \\
\text { Degree }\end{array}$ & $\begin{array}{c}\text { nOut } \\
\text { Degree }\end{array}$ & $\begin{array}{c}\text { nBet } \\
\text { weenness }\end{array}$ & $\begin{array}{c}\text { k- } \\
\text { Cores }\end{array}$ \\
\hline IESE Business School & iese.edu & 42,86 & 4,08 & 3,04 & 21 \\
\hline La Salle & salleurl.edu & 16,33 & 4,08 & 2,02 & 10 \\
\hline Univ. Nacional Educación a Distancia & uned.es & 16,33 & 42,86 & 1,24 & 21 \\
\hline Universidad Autónoma de Madrid & uam.es & 8,16 & 48,98 & 0,64 & 21 \\
\hline Universidad Carlos III de Madrid & uc3m.es & 46,94 & 26,53 & 0,77 & 21 \\
\hline Universidad Complutense de Madrid & ucm.es & 14,29 & 77,55 & 3,49 & 21 \\
\hline Universidad de A Coruña & udc.es & 77,55 & 30,61 & 9,01 & 21 \\
\hline Universidad de Alcalá de Henares & uah.es & 51,02 & 12,24 & 1,57 & 21 \\
\hline Universidad de Almería & ual.es & 57,14 & 14,29 & 2,85 & 21 \\
\hline Universidad de Cádiz & uca.es & 55,10 & 6,12 & 1,38 & 21 \\
\hline Universidad de Cantabria & unican.es & 46,94 & 4,08 & 0,65 & 21 \\
\hline Universidad de Castilla-La Mancha & uclm.es & 32,65 & 18,37 & 1,31 & 21 \\
\hline Universidad de Córdoba & uco.es & 16,33 & 12,24 & 0,54 & 13 \\
\hline Universidad de Deusto & deusto.es & 4,08 & 6,12 & 0,00 & 5 \\
\hline Universidad de Extremadura & unex.es & 6,12 & 24,49 & 2,21 & 12 \\
\hline Universidad de Granada & ugr.es & 14,29 & 83,67 & 4,84 & 21 \\
\hline Universidad de Huelva & uhu.es & 12,24 & 8,16 & 0,14 & 10 \\
\hline Universidad de Jaén & ujaen.es & 8,16 & 16,33 & 0,58 & 11 \\
\hline Universidad de La Laguna & ull.es & 8,16 & 44,90 & 0,33 & 21 \\
\hline Universidad de La Rioja & unirioja.es & 8,16 & 2,04 & 2,02 & 5 \\
\hline Univ. de Las Palmas de Gran Canaria & ulpgc.es & 55,10 & 6,12 & 0,77 & 21 \\
\hline Universidad de León & unileon.es & 18,37 & 4,08 & 0,61 & 10 \\
\hline Universidad de Málaga & uma.es & 16,33 & 40,82 & 2,04 & 21 \\
\hline Universidad de Murcia & um.es & 14,29 & 42,86 & 0,03 & 21 \\
\hline Universidad de Navarra & unav.es & 32,65 & 8,16 & 1,74 & 19 \\
\hline Universidad de Oviedo & uniovi.es & 12,24 & 26,53 & 0,82 & 19 \\
\hline Universidad de Salamanca & usal.es & 8,16 & 28,57 & 0,48 & 18 \\
\hline Univ. de Santiago de Compostela & usc.es & 44,90 & 51,02 & 5,49 & 21 \\
\hline Universidad de Sevilla & us.es & 42,86 & 59,18 & 7,82 & 21 \\
\hline Universidad de Valladolid & uva.es & 28,57 & 36,73 & 0,52 & 21 \\
\hline Universidad de Vigo & uvigo.es & 63,27 & 26,53 & 3,69 & 21 \\
\hline Universidad de Zaragoza & unizar.es & 42,86 & 36,73 & 1,20 & 21 \\
\hline Universidad del País Vasco & ehu.es & 38,78 & 44,90 & 4,49 & 21 \\
\hline Universidad Miguel Hernández & umh.es & 44,90 & 4,08 & 0,66 & 21 \\
\hline Universidad Politécnica de Madrid & upm.es & 4,08 & 59,18 & 2,24 & 21 \\
\hline Universidad Pontificia de Comillas & upco.es & 10,20 & 2,04 & 1,10 & 6 \\
\hline Universidad Pública de Navarra & unavarra.es & 10,20 & 2,04 & 0,35 & 6 \\
\hline Universidad Rey Juan Carlos & urjc.es & 57,14 & 8,16 & 1,95 & 21 \\
\hline Universitat Autònoma de Barcelona & uab.es & 2,04 & 63,27 & 1,76 & 21 \\
\hline Universitat d'Alacant & ua.es & 4,08 & 97,96 & 5,11 & 21 \\
\hline Universitat de Barcelona & ub.es & 8,16 & 53,06 & 0,87 & 21 \\
\hline Universitat de Girona & udg.es & 10,20 & 14,29 & 0,11 & 12 \\
\hline Universitat de les Illes Balears & uib.es & 67,35 & 22,45 & 1,77 & 21 \\
\hline Universitat de Lleida & udl.es & 18,37 & 22,45 & 2,40 & 18 \\
\hline Universitat de Valencia & uv.es & 42,86 & 0,00 & 0,00 & 18 \\
\hline Universitat Jaume I de Castelló & uji.es & 24,49 & 30,61 & 0,41 & 21 \\
\hline Universitat Oberta de Catalunya & uoc.edu & 16,33 & 12,24 & 4,68 & 12 \\
\hline Universitat Politècnica de Catalunya & upc.es & 44,90 & 46,94 & 5,30 & 21 \\
\hline Universitat Politècnica de Valencia & upv.es & 22,45 & 48,98 & 3,83 & 21 \\
\hline Universitat Pompeu Fabra & upf.es & 55,10 & 16,33 & 1,41 & 21 \\
\hline
\end{tabular}

Apéndice 1. Medidas de centralidad y propiedades de las 50 universidades españolas en la web académica española 


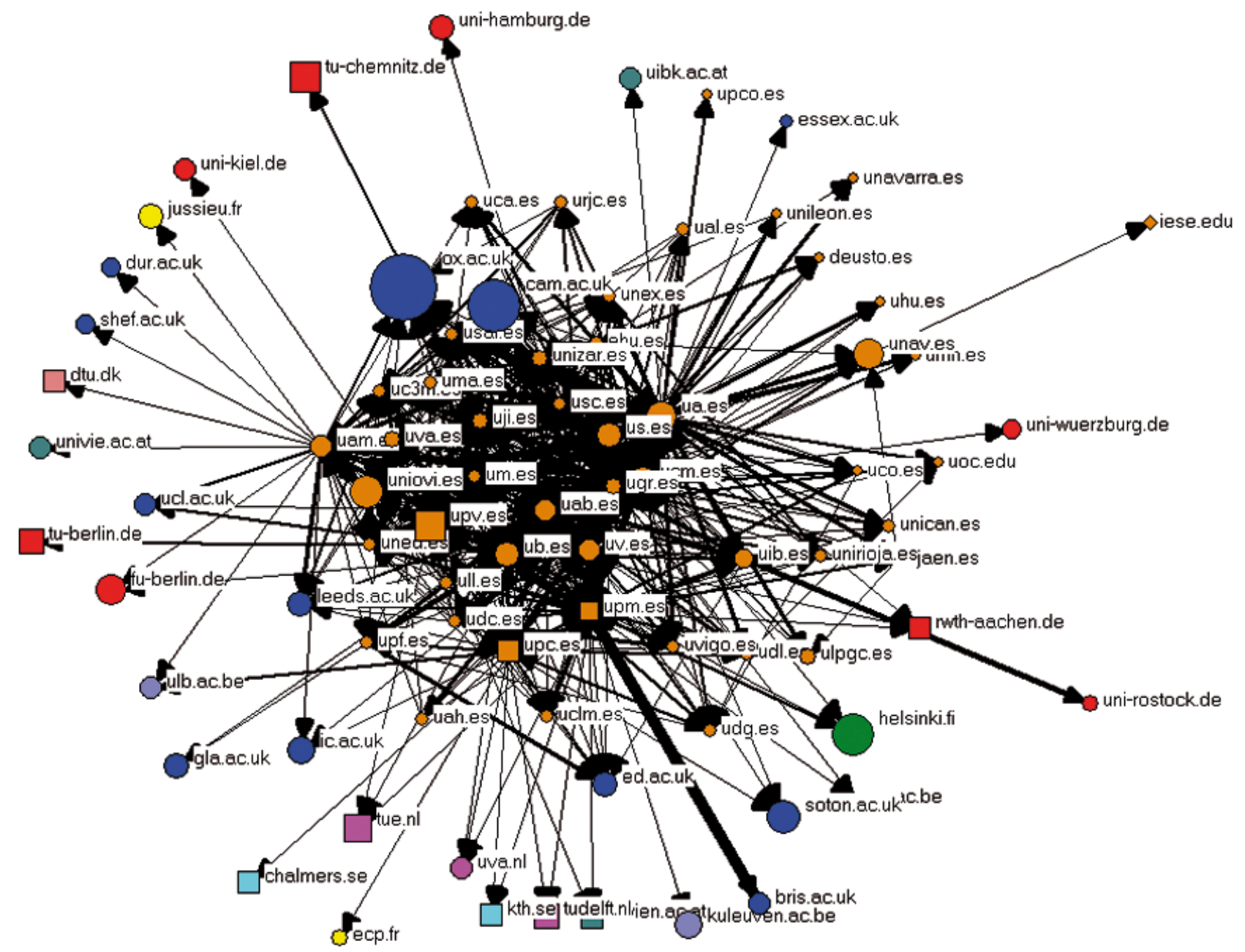

Figura 3. Mapa de enlaces salientes de las universidades españolas ( 85 nodos; $\operatorname{arcos} \geq 50$ enlaces; layout: Spring embedding)

necientes a regiones diferentes como es el caso de la Universitat de les Illes Balears (uib.es) y la Universitat Jaume I de Castelló (uji.es). Quizás España es el país donde mejor se vean las relaciones temáticas de universidades tecnológicas (politécnicas), distanciándose en este punto la de Valencia (upv.es), Madrid (upm.es) y Cataluña (upc.es), representadas con forma de cuadrados. Es significativo apreciar también la escasa presencia que tienen los centros privados, que se encuentran en una posición bastante periférica respecto al resto de universidades, a excepción de la Universitat Oberta de Catalunya (uoc.es), quizás debido al uso intensivo de la web como medio docente y campo de investigación. Este dato también es apuntado por Gómez, Bordons, Fernández y Morillo (2007) desde un punto de vista cienciométrico.

Como se ha comentado anteriormente, la web española muestra una red bastante compacta. El análisis de $k$-cores (apéndice 1) confirma esta percepción al detectar un grupo central de 31 universidades $(62 \%)$ con un grado 21, lo que corrobora esta alta cohesión. Dicho grupo lo forman universidades públicas con una asentada trayectoria en el panorama educativo español.

En cuanto al grado de la red (apéndice 1), las uni- versidades con un mayor grado de entrada normalizado (nInDegree) son la de A Coruña (udc.es) $(77,55)$, Illes Balears $(67,35)$ y Vigo (uvigo.es) $(63,27)$. Las que cuentan con un mayor grado de salida normalizado (nOutDegree) son Alicante $(97,96)$, Granada (ugr. es) $(83,67)$ y Complutense de Madrid (ucm.es) $(77,55)$. Todas pertenecen al grupo central comentado anteriormente, así el grado de entrada y salida no nos informa mucho sobre la estructura de la red. Esto viene reforzado por el grado de intermediación normalizado (nBetweenness), cuyo valor más alto lo posee $A$ Coruña $(9,01)$ y que muestra un escaso porcentaje de intermediación, siendo éste un indicador de redes compactas y homogéneas.

En el gráfico de enlaces salientes españoles (figura 3) hacia el resto de universidades europeas, se aprecia con sorpresa que, para el número y el tamaño de las universidades españolas, éstas citen a tan pocas europeas del grupo principal, tan sólo 35 centros europeos $(6,64 \%)$. Este hecho se aprecia también en el gráfico europeo (figura 1), donde la red española aparece bastante aislada del resto de espacios web europeos. La Tabla 1 muestra las cinco universidades españolas que más enlazan a universidades europeas. Nótese que al- 


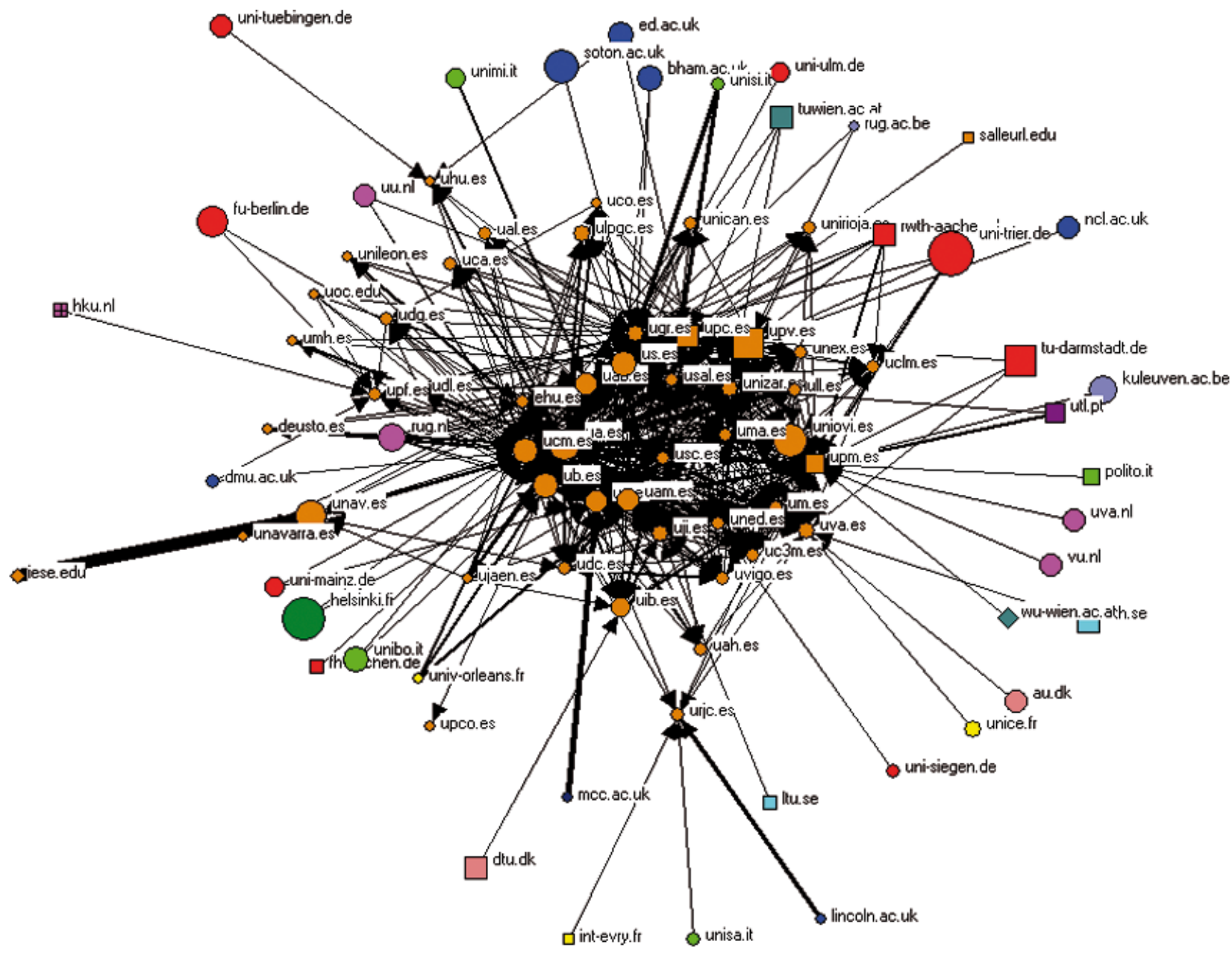

Figura 4. Mapa de enlaces entrantes de las universidades españolas (89 nodos; arcos $\geq 50$ enlaces; layout: Spring embedding)

gunas de éstas son enlazadas por más de una española, por lo que el Total no debe entenderse como sumatorio de webs enlazadas. Las que más citan a universidades europeas son la Universidad Politécnica de Madrid (42,86\%) y la Autónoma de Madrid (31,42\%).

En el mapa de enlaces entrantes (figura 4) existe ligeramente una mayor cantidad de universidades que citan a las españolas, aunque el número sigue siendo muy inferior (39 universidades, 7,4\%). La Tabla 2 muestra las cinco universidades españolas que son más enlazadas desde Europa, destacando la Politécnica de Madrid $(27,27 \%)$ y la de Granada $(24,24 \%)$. Al igual que en el gráfico de enlaces salientes, se encuentra un escaso número de universidades europeas que citan a las españolas, con un promedio de $46 \%$ de universidades españolas citadas desde Europa. Tanto en la figura 3 como en la 4, se aprecia que las universidades politécnicas son las que poseen una mayor presencia europea.

En el gráfico radial (figura 5), se observa cómo las universidades españolas citan casi en exclusiva a las británicas $(62,63 \%)$, mientras que las europeas que dirigen sus enlaces a las españolas provienen de Alemania $(28,27 \%)$ y Países Bajos $(20,55 \%)$.

\begin{tabular}{|l|l|c|c|c|}
\hline & Universidad & URL & $\begin{array}{c}\text { Webs } \\
\text { enlazadoras }\end{array}$ & $\%$ \\
\hline 1 & $\begin{array}{l}\text { Universidad } \\
\text { Politécnica de } \\
\text { Madrid }\end{array}$ & upm.es & 9 & 27,27 \\
\hline 2 & $\begin{array}{l}\text { Universidad de } \\
\text { Granada }\end{array}$ & ugr.es & 8 & 24,24 \\
\hline 3 & $\begin{array}{l}\text { Universidad } \\
\text { Complutense } \\
\text { de Madrid }\end{array}$ & ucm.es & 7 & 21,21 \\
\hline 4 & $\begin{array}{l}\text { Universitat de } \\
\text { Barcelona }\end{array}$ & ub.es & 5 & 15,15 \\
\hline 5 & $\begin{array}{l}\text { Universitat } \\
\text { Politècnica de } \\
\text { Catalunya }\end{array}$ & upc.es & 4 & 12,12 \\
\hline & Total & 39 & 100,00 \\
\hline
\end{tabular}

Tabla 2. Universidades españolas que son enlazadas por universidades europeas

\section{Discusión}

Uno de los principales temas a discutir en un análisis de enlaces es la significación de éstos. Wilkinson, Harries, Thelwall y Price (2003) encontraron eviden- 


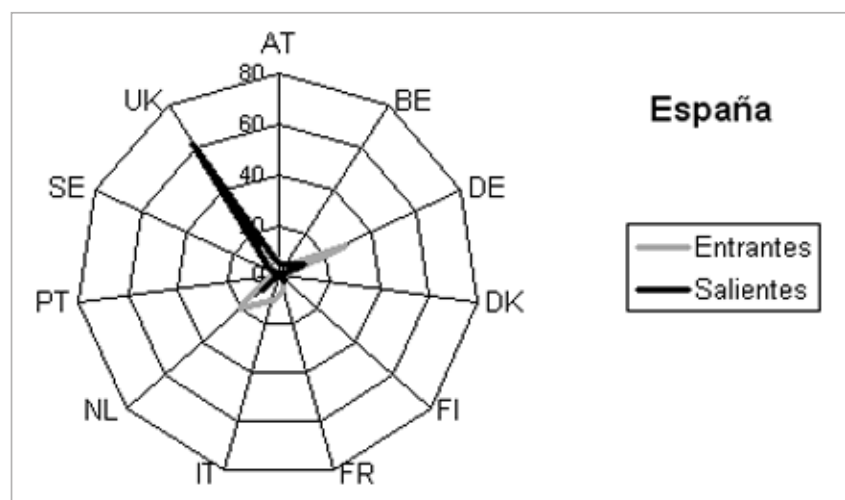

Figura 5. Origen de las universidades citantes y citadas de la red española

cias de que casi el $90 \%$ de los enlaces entre universidades estaban relacionados con la actividad académica. También Thelwall y Harries (2003) encontraron una fuerte correlación entre la cantidad de enlaces entrantes de universidades británicas y su valoración académica (Research Assessment Exercise). Esto nos permite pensar que los enlaces son objetos de análisis de alto valor por la significación académica y científica que tienen en la web universitaria. Pero también es lógico pensar que el desarrollo web de una universidad no tiene por qué ir parejo a la actividad global de la misma, aunque sí da muestras de su desarrollo tecnológico.

Sin embargo, uno de los grandes problemas ha sido la consistencia de los buscadores como herramienta para la obtención de enlaces. Diversos estudios han encontrado distintos problemas con la cobertura (Lawrence; Giles, 1998; Vaughan; Thelwall, 2004), inestabilidad (Bar-Ilan, 1998; Rousseau, 1998) e inconsistencia de los operadores (Ingwersen, 1998). Nuestra dificultad con la Universidad de Valencia es una muestra de ello. Esto nos obliga a ser cautos con los resultados ya que las relaciones encontradas dependen de la información suministrada por los buscadores. Aun así pensamos que estas herramientas son la fuente más apropiada para estudios de gran envergadura ya que su coste en tiempo y recursos es mucho menor que otras fuentes de datos como pueden ser los crawlers o robots.

\section{Conclusiones}

El análisis de las distintas redes de enlaces y la visualización del espacio académico europeo nos permite concluir que la red española está bastante aislada del resto de Europa y que salvo las politécnicas, la relación con el resto de universidades europeas es muy escasa.

En el gráfico europeo, la red española se encuentra aislada por su bajo grado de co-enlaces, ya que tan sólo el 7,4\% de las universidades europeas enlazan a las españolas. Una de las causas de este hecho puede deberse al escaso uso de contenidos en inglés por la comunidad académica española, lo que hace que sea poco enlazada y muestre una posición aislada con respecto al resto de universidades europeas (Ortega, et al., 2008). Por ello, pensamos que las universidades españolas deberían adoptar una política más internacional de publicación de contenidos web, incrementando el número de páginas en inglés y de contenidos de calidad como repositorios de publicaciones científicas. Todo esto permitiría una mayor integración de las universidades españolas dentro de la red universitaria europea.

\section{"Las universidades españolas deberían adoptar una política de publicación de contenidos web más internacional, incrementando el número de páginas en inglés"}

Respecto a la propia red académica española, las distintas medidas del ARS han confirmado lo compacta y homogénea que es, al detectar un grupo central muy conectado de 31 universidades. Por esto, las medidas de centralidad (grado nodal y de intermediación) no han podido detectar universidades que destaquen dentro de la red, ni que tengan un papel relevante en su cohesión. Tampoco se han identificado universidades pasarela que mantengan fuertes vínculos con el resto de universidades europeas, lo que provoca que la red española sea poco accesible desde otras universidades de Europa.

La red española muestra una fuerte vinculación hacia el Reino Unido, mientras que las universidades que citan a la red española provienen mayoritariamente de Alemania y Países Bajos. Este patrón es similar con otros países ya que Reino Unido es el país que más enlaces recibe, mientras que Alemania es el que más realiza (Ortega, et al., 2008).

\section{Referencias}

Aguillo, I. F. Cybermetrics. Madrid: Cindoc, CSIC, 1997. http://www.cindoc.csic.es/cybermetrics/

Aguillo, I. F.; Granadino, B.; Ortega, J. L.; Prieto, J. A. "Scientific research activity and communication measured with cybermetrics indicators". En: Journal of the American Society for Information Science and Technology, 2006, v. 57, n. 10, pp. 1.296-1.302.

Alonso-Berrocal, J. L.; García-Figuerola, L. C.; Zazo-Rodríguez, F. Cibermetría: nuevas técnicas de estudio aplicables al web. Madrid: Trea, 2004. ISBN 8497041143.

Baeza-Yates, R.; Castillo, C.; López, V. "Characteristics of the web of Spain”. En: Cybermetrics, 2005, v. 9, n. 1.

http://www.cindoc.csic.es/cybermetrics/articles/v9ilp3.html

Barabási, A. L.; Albert, R.; Jeong, H. "Scale-free characteristics of random networks: the topology of the world-wide web". En: Physica a, 2000, v. 281 , n. $1-4$, pp. $69-77$.

Bar-Ilan, J. "Search engine results over time - a case study on search engine 
stability". En: Cybermetrics, 1998, v. 1, paper 1.

http://www.cindoc.csic.es/cybermetrics/articles/v2ilpl.html

Barro-Ameneiro, S. (ed.); Fernández-López, S.; Rodeiro-Pazos, D. Ruzo-Sanmartín, E.; Canay-Pazos, R.; Franco-Tubío, J. Las tecnologías de la información y las comunicaciones en el sistema universitario español. Madrid: CRUE, 2004. ISBN 8493278335

http://www.crue.org/pdf/TIC.pdf

Dodge, M. Cyber-geography research. Consultado en: 20-04-07. http://www.cybergeography.org/home.html

Faba-Pérez, C.; Guerrero-Bote, V. P.; Moya-Anegón, F. Fundamen tos y técnicas cibermétricas. Mérida: Junta de Extremadura, 2004. ISBN 8496212173

Gómez, I.; Bordons, M.; Fernández, M. T.; Morillo, F. "Structure and research perfomance of Spanish universities". En: Proceeding of the $11^{\text {th }} \mathrm{In}$ ternational Conference of the International Society for Scientometrics and Informetrics, 2007 (en prensa).

Heimeriks, G.; Horlesberger, M.; Van Den Besselaar, P. "Mapping communication and collaboration in heterogeneous research networks". En: Scientometrics, 2003 , v. 58, n. 2, pp. 391-413

Heimeriks, G.; Van Den Besselaar, P. "Analyzing hyperlinks networks the meaning of hyperlink based indicators of knowledge production". En Cybermetrics, 2006, v. 10, n. 1, paper 1.

http://www.cindoc.csic.es/cybermetrics/articles/v10i1p1.html

Ingwersen, P. "The calculation of web impact factors". En: Journal of documentation, 1998, v. 54, n. 2, pp. 236-243.

Lawrence, S.; Giles, C. L. "Accessibility of information on the web". En Nature, 1999, n. 400, v. 6.740, pp. 107-109.

Leydesdorff, L. "Betweenness centrality" as an indicator of the "interdisciplinarity" of scientific journals". En: Journal of the American Society for Information Science and Technology, 2007 (en prensa).

http://users.fmg.uva.nl/lleydesdorff/betweenness/index.htm

Nooy, W. d.; Mrvar, A.; Batagelj, V. Exploratory social network analysis with pajek. Cambridge, UK: Cambridge University Press, 2005. ISBN 0521602629

Ortega, J. L. Visualización de la web universitaria europea: análisis cuantitativo de enlaces a través de técnicas cibermétricas. Madrid: Universidad Carlos III de Madrid, 2007

Ortega, J. L.; Aguillo, I. F. “Análisis de co-enlaces: una aproximación teórica”. En: El profesional de la información, 2006, v. 15, n. 4, pp. 270-277.

Ortega, J. L.; Aguillo, I. F.; Cothey, V.; Scharnhorst, A. "Maps of the academic web in the European Higher Education Area - an exploration of visual web indicators". En: Scientometrics, 2008, v. 74, n. 2.

http://internetlab.cindoc.csic.es/cv/11/EU_Web_maps/EU_Web_maps.htm

Ortega, J. L.; Aguillo, I. F. "Linear analysis of cybermetric data: quantifying the European university web space". En: Proceedings of the $11^{\text {th }}$ In ternational Conference of the International Society for Scientometrics and Informetrics, 2007.

Paterson, R.; Cox, D. Visualization study of the Nsfnet. Consultado en: 20 04-07.

http://vis.ncsa.uiuc.edu/?content $=$ projects \&subcontent $=$ show $\& I D=4$

Pinto-Molina, M.; Alonso-Berrocal, J. L.; Cordón-García, J. A.;
Fernández-Marcial, V.; García-Figuerola, C.; García-Marco, J.; Gómez-Camarero, C.; Zazo, Á. F.; Doucet, A. V. "Análisis cualitativo de la visibilidad de la investigación de las universidades españolas a través de sus páginas web". En: Revista española de documentación científica, 2004, v. 27 , n. 3, pp. 345-370.

Polanco, X.; Boudourides, M.; Besagni, D.; Roche, I. "Clustering and mapping european university web sites sample for displaying associations and visualizing networks". En: NTTS\&ETK 2001 Conference, 2001.

Rodríguez-Gairín, J. M. "Valoración del impacto de la información en internet: Altavista, el "citation index" de la red". En: Revista española de documentación científica, 1997, v. 20, n. 2, pp. 175-181.

Rousseau, R. "Daily time series of common single word searches in AltaVista and NorthernLight". En: Cybermetrics, 1998, n. 1, paper 2. http://www.cindoc.csic.es/cybermetrics/articles/v2ilp2.html

Sanz-Menéndez, L. "Análisis de redes sociales: o como representar las estructuras sociales adyacentes". En: Apuntes de ciencia y tecnología, 2003, n. 7, pp. 21-29.

Seidman, S. B. "Network structure and minimum degree". En: Social networks, 1983 , v. 5, pp. 269-287.

Térmens-Graells, M.; Ribera-Turró, M.; Sulé-Duesa, A. "Nivel de accesibilidad de las sedes web de las universidades españolas". En: Revista española de documentación científica, 2003, v. 26, n. 1, pp. 21-39.

Thelwall, M.; Aguillo, I. F. "La salud de las web universitarias españolas". En: Revista española de documentación científica, 2003, v. 26, n. 3, pp 291-305.

http://www.eicstes.org/EICSTES_PDF/PAPERS/La salud de las Web universitarias españolas (Thelwall-Aguillo).PDF

Thelwall, M.; Harries, G. "The connection between the research of a university and counts of links to its web pages: an investigation based upon a classification of the relationships of pages to the research of the host university". En: Journal of the American Society for Information Science and Technology, 2003, v. 54, n. 7, pp. 594-602.

Vaughan, L. "Visualizing linguistic and cultural differences using web colink data". En: Journal of the American Society for Information Science and Technology, 2006, v. 57, n. 9, pp. 1.178-1.193.

Vaughan, L.; Thelwall, M. "Search engine coverage bias: evidence and possible causes". En: Information processing and management: an international journal, 2004, v. 40, n. 4, pp. 693-707

Wilkinson, D.; Harries, G.; Thelwall, M.; Price, E. "Motivations for academic web site interlinking: evidence for the web as a novel source of information on informal scholarly communication". En: Journal of information science, 2003, v. 29, n. 1, pp. 95-111.

Zook, M. A. "The web of production: the economic geography of commercial internet content production in the United States". En: Environment and planning A, 2000, v. 32, pp. 411-426.

José-Luis Ortega, Isidro F. Aguillo, Laboratorio de Cibermetría, Cindoc, CSIC, Joaquín Costa, 22. 28002 Madrid. jortega@cindoc.csic.es isidro@cindoc.csic.es

\section{PROMOCIÓN PARA NUEVOS SUSCRIPTORES DE EPI}

Si te suscribes a El profesional de la información entre ahora y Navidad de 2007

te regalamos un Anuario ThinkEPI 2007.

Menciona esta oferta cuando te suscribas

a través del formulario de suscripción online o del boletín en papel. 
Scopus es la mayor base de datos de resúmenes y citas de la literatura revisada por expertos y fuentes web de calidad con herramientas inteligentes para buscar, analizar y visualizar la investigación.

\section{Enriquezca su experiencia Refine su investigación \\ - 15.000 publicaciones periódicas revisadas por expertos de más de 4.000 editores internacionales. \\ - Más de 1.000 publicaciones de libre acceso \\ - 500 actas de congreso \\ - Más de 125 series monográficas \\ - Más de 600 publicaciones comerciales \\ - 33 millones de registros, de los cuales: \\ - 16 millones incluyen referencias que se remontan a 1996 \\ - 17 millones son anteriores a 1996, y se remontan a 1869}

Scopus también abarca 386 millones de páginas web ciehtíficas, incluyendo 21 millones de patentes

Acceso de prueba disponible en: www.scopus.com 